\title{
The Genome of the CTG(Ser1) Yeast Scheffersomyces stipitis Is Plastic
}

\author{
Samuel Vega-Estévez, ${ }^{\text {a }}$ Andrew Armitage, ${ }^{\text {b Helen J. Bates, }, ~ R i c h a r d ~ J . ~ H a r r i s o n, ~}{ }^{c}$ (D) Alessia Buscainoa \\ anniversity of Kent, School of Biosciences, Kent Fungal Group, Canterbury Kent, United Kingdom \\ bNatural Resources Institute, University of Greenwich, Chatham Maritime, Kent, United Kingdom \\ cNIAB Cambridge Crop Research, Cambridge, United Kingdom
}

ABSTRACT Microorganisms need to adapt to environmental changes, and genome plasticity can lead to rapid adaptation to hostile environments by increasing genetic diversity. Here, we investigate genome plasticity in the CTG(Ser1) yeast Scheffersomyces stipitis, an organism with an enormous potential for second-generation biofuel production. We demonstrate that $S$. stipitis has an intrinsically plastic genome and that different S. stipitis isolates have genomes with distinct chromosome organizations. Real-time evolution experiments show that $S$. stipitis genome plasticity is common and rapid since extensive genomic changes with fitness benefits are detected following in vitro evolution experiments. Hybrid MinION Nanopore and Illumina genome sequencing identify retrotransposons as major drivers of genome diversity. Indeed, the number and position of retrotransposons are different in different S. stipitis isolates, and retrotransposon-rich regions of the genome are sites of chromosome rearrangements. Our findings provide important insights into the adaptation strategies of the CTG(Ser1) yeast clade and have critical implications in the development of second-generation biofuels. These data highlight that genome plasticity is an essential factor for developing sustainable S. stipitis platforms for second-generation biofuels production.

IMPORTANCE Genomes contain genes encoding the information needed to build the organism and allow it to grow and develop. Genomes are described as stable structures where genes have specific positions within a chromosome. Changes in gene dosage and position are viewed as harmful. However, it is becoming increasingly clear that genome plasticity can benefit microbial organisms that need to adapt rapidly to environmental changes. Mechanisms of genome plasticity are still poorly understood. This study focuses on Scheffersomyces stipitis, a yeast that holds great potential for second-generation biofuel production generated from forestry and agriculture waste. We demonstrate that $S$. stipitis chromosomes are easily reshuffled and that chromosome reshuffling is linked to adaptation to hostile environments. Genome sequencing demonstrates that mobile genetic elements, called transposons, mediate $S$. stipitis genome reshuffling. These data highlight that understanding genome plasticity is important for developing sustainable S. stipitis platforms for second-generation biofuels production.

KEYWORDS biofuels, CTG clade yeast, genome plasticity, genomic instability, transposons

\footnotetext{
- ukaryotic genomes are often described as stable structures with well-preserved chromosome organization, and genome instability is viewed as harmful. However, an increasing body of evidence demonstrates that eukaryotic microorganisms have a plastic genome and genome instability is instrumental for rapid and reversible adaptation to hostile environments (1-4). This is because genomic instability can increase genetic diversity, allowing the selection of genotype(s) better adapted to a new environment $(5,6)$. Repetitive DNA elements are major contributors to genome plasticity since repeats can undergo inter- and intralocus recombination, resulting in gene conversion, gross chromosomal rearrangements,
}

Citation Vega-Estévez S, Armitage A, Bates $\mathrm{H}$ Harrison RJ, Buscaino A. 2021. The genome of the CTG(Ser1) yeast Scheffersomyces stipitis is plastic. mBio 12:e01871-21. https://doi.org/10 $.1128 / \mathrm{mBio} .01871-21$

Editor Judith Berman, Tel Aviv University Copyright $\odot 2021$ Vega-Estévez et al. This is an open-access article distributed under the terms of the Creative Commons Attribution 4.0 International license. Address correspondence to Alessia Buscaino, A.Buscaino@kent.ac.uk.

Received 6 July 2021

Accepted 5 August 2021

Published 7 September 2021 
and segmental aneuploidies (7). Transposable elements (TEs), a specific class of repetitive elements, alter genome organization by recombination-dependent mechanisms and by jumping to new sites in the genome (8). TEs belong to two major classes: DNA transposons (class II) and retrotransposons (class I). DNA transposons utilize a "cut and paste" mechanism in which the parental element excises from its original location before integrating elsewhere (9). In contrast, retrotransposons replicate through reverse transcription of their RNA and integrate the resulting cDNA into another locus. Retrotransposons can be further classified into long-terminal-repeat (LTR) retrotransposons and non-LTR retrotransposons (10). LTR retrotransposons are characterized by two LTR sequences flanking an internal coding region containing the genes encoding for the structural protein GAG and enzyme POL required for reverse transcription and integration (11). While POL enzymes are conserved across organisms, GAG proteins are poorly conserved (12). LINE elements are one of the most abundant non-LTR retrotransposons, and they are typically composed of a $5^{\prime}$-noncoding region, two open reading frames (ORF1 and ORF2) and a 3 '-noncoding region that is marked by a poly(A) tail (13). ORF1 proteins have a diverse amino acid sequence, but they often contain a DNA-binding motif (14). ORF2 encodes endonuclease and reverse transcriptase activity that is critical for transposition (15).

The CTG(Ser1) clade of fungi, in which the CTG codon is translated as serine rather than leucine, is an important group of ascomycetous yeasts featuring those that hold great promise in biotechnology, such as Scheffersomyces stipitis, and dangerous human fungal pathogens, such as Candida albicans (16). The CTG(Ser1) clade comprises several species with different lifestyles and genomic organizations, including haploid and diploid species that colonize diverse environments by reproducing sexually or para-sexually (16-19). One common feature of CTG(Ser1) species is their ability to adapt remarkably well to extreme environments (20). For example, CTG(Ser1) yeasts can grow on various carbon sources and are highly tolerant to environmental changes such as changes in osmolarity $(16,19,20)$. It is well established that genome plasticity is a critical adaptive mechanism in the human fungal pathogens Candida albicans, the most studied CTG(Ser1) clade member (4). In C. albicans, stress increases genome instability by affecting the rate and type of genomic rearrangements (21). Different classes of DNA repeats drive this genetic variation, including TEs, long repeats and major repeat sequences (MRS) (22-24). It is still unknown whether genome plasticity is a general feature of the $C T G(\operatorname{Ser} 1)$ clade and whether DNA repeats are drivers for genome diversity across this yeast group.

This study investigates genome plasticity in S. stipitis, a CTG(Ser1) clade yeast with great potential for the eco-friendly and ethical production of second-generation biofuels (2527). Second-generation biofuels are generated by fermentation of lignocellulose biomass, produced in large amounts ( $>1.3$ billion tons produced annually) as waste following agricultural and forestry processing operations (27). Lignocellulose is a heteropolymer composed of fermentable hexose sugars (i.e., glucose) and pentose sugars (i.e., xylose) (28). The yeast Saccharomyces cerevisiae, usually the organism of choice for industrial production of ethanol, is not suitable for producing second-generation ethanol because it cannot ferment pentose sugars as it lacks specific transporters enzymatic network important for their metabolism (28). S. stipitis holds excellent potential for biofuel derived from green waste because it is one of the few yeast species that can ferment both hexose and pentose sugars (25-27). S. stipitis is a nonpathogenic haploid yeast that is found in the gut of wood-ingesting beetles, in hardwood forests or areas high in agricultural waste (29). Contrary to C. albicans, S. stipitis has a canonical sexual cycle whereby mating of haploid cells generate diploid cells that undergo meiosis and produce haploid spores (30). Although several S. stipitis natural isolates are used for the optimization of second-generation biofuels production, the genome of only one strain (Y-11545) has been sequenced and assembled to the chromosomal level (31). The Y-11545 genome has a size of 15.4 million base pairs (Mbp) organized in eight chromosomes and containing $\sim 6,000$ protein-coding genes (31-33). S. stipitis chromosomes are marked by regional centromeres composed of full-length LTR retrotransposons (Tps5a, Tps5b, and Tps5c) and noncoding, nonautonomous LARD (large retrotransposon derivative) elements $(31,33)$.

To investigate the plasticity of the $S$. stipitis genome, we have taken several complementary approaches. First, we systematically identified S. stipitis DNA repeats and investigated 
the genotypic diversity of 27 different S. stipitis natural isolates collected from different environments. Second, we combined MinION Nanopore with Illumina genome sequencing to generate a high-quality chromosome-level sequence assembly of a second $S$. stipitis natural isolate (Y-7124) and compared its genome structure to the reference Y-11545 genome. Lastly, we performed in vitro evolution experiments and analyzed S. stipitis genome organization changes following laboratory passaging under stress or unstressed growth conditions. Thanks to this combined approach, we discovered that the S. stipitis genome is plastic. We demonstrate that different $S$. stipitis natural isolates have distinct chromosomal organizations and that transposable elements drive this extensive intra-species genetic variation. Genome plasticity is not regulated by stress; however, large chromosome rearrangements are linked to adaptation to hostile environments. Our findings have important implications for secondgeneration biofuel production as genome plasticity is a paramount factor to be considered for the successful development of superior biofuel-producer S. stipitis strains.

\section{RESULTS}

Classification of S. stipitis DNA repeats. DNA repeats are drivers of genome variation. Comparative genomic analyses have identified different repetitive elements in some CTG (Ser1) clade members, yet a comprehensive survey of $S$. stipitis repetitive elements is lacking $(18,34)$. Therefore, we sought to classify the major classes of repetitive elements associated with the Y-11545 sequenced genome by aligning the genomic sequence of each strain to itself and identifying long sequences ( $>100$ nucleotides [nt]) present more than once in the genome. The genomic position of these repeats was manually verified, and clustered repeats were combined and categorized depending on their genomic positions, structures, and sequence similarity. Our analyses identified known S. stipitis repeat-rich loci such as centromeric transposon clusters, the NUPAV sequence, an integrated L-A dsRNA virus, and several gene families $(32,33,35)$. As observed in other members of the CTG(Ser1) clade, we did not detect any MRS, a class of repetitive elements found only in C. albicans and the closely related $C$. dubliniensis and $C$. tropicalis species $(18,34)$. Here we focus on intra- or interchromosomal repeats that have not been described to date: noncentromeric TEs, subtelomeric regions, and telomeric repeats (Fig. 1).

We identified six novel retrotransposon families scattered along chromosome arms: three LTR retrotransposons (Ava, Bea, and Caia) and three LINE retrotransposons (Ace, Bri, and (an) (Fig. 1A; see also Table S3 in the supplemental material). Ava, Bea, and Caia have a similar structure where two identical LTR sequences flank an internal domain. The internal domain contains two ORFs: one encoding for a putative POL and one encoding for an S. stipitis-specific protein that we named LTR-associated protein (Lap1 in Ava, Lap2 in Bea, and Lap3 in Caia). Homology search failed to identify any GAG gene associated with the Ava, Bea, and Caia retrotransposons. Since Gag proteins are poorly conserved among different organisms, we hypothesize that the Lap proteins are Gag proteins.

Ace, Bri, and Can are LINE elements composed of the noncoding regions NC-1 and NC-2 surrounding an internal coding region encoding for a Pol enzyme and an S. stipitis-specific LINE-associated protein (Linea1 in Ace, Linea2 in Bri, and Linea3 in Can). Linea1 and Linea2, but not Linea 3, have a zinc-finger DNA-binding motif (see Table S3). Comparison across the CTG(Ser1) clade revealed that S. stipitis TE repertoire is typical of this clade. Indeed, retrotransposons are common in this yeast group: the genome of all species analyzed contains LTR elements, whereas LINE elements are present in 6/8 species (Fig. 1B; see also Table S4). Similarly to other CTG(Ser1) clade yeasts, we did not detect any DNA transposons integrated into the S. stipitis genome (Fig. 1B; see also Table S4).

Our repeat analysis demonstrates that the terminal sequences of $S$. stipitis chromosomes are repeat-rich and composed of two elements with different degrees of repetitiveness: telomere proximal repeats and subtelomeric regions. The telomeric repeats are noncanonical and composed of 24-nucleotide units repeated in tandem. Each unit contains a TG motif reminiscent of typical telomeric repeats (Fig. 1C). S. stipitis subtelomeric regions (the $\sim 30$-kb region adjacent to telomeric repeats) are enriched in retrotransposon-derived elements. Indeed, DNA sequences with homology to Bea LTR retrotransposons and Ace LINE elements are found in 
A

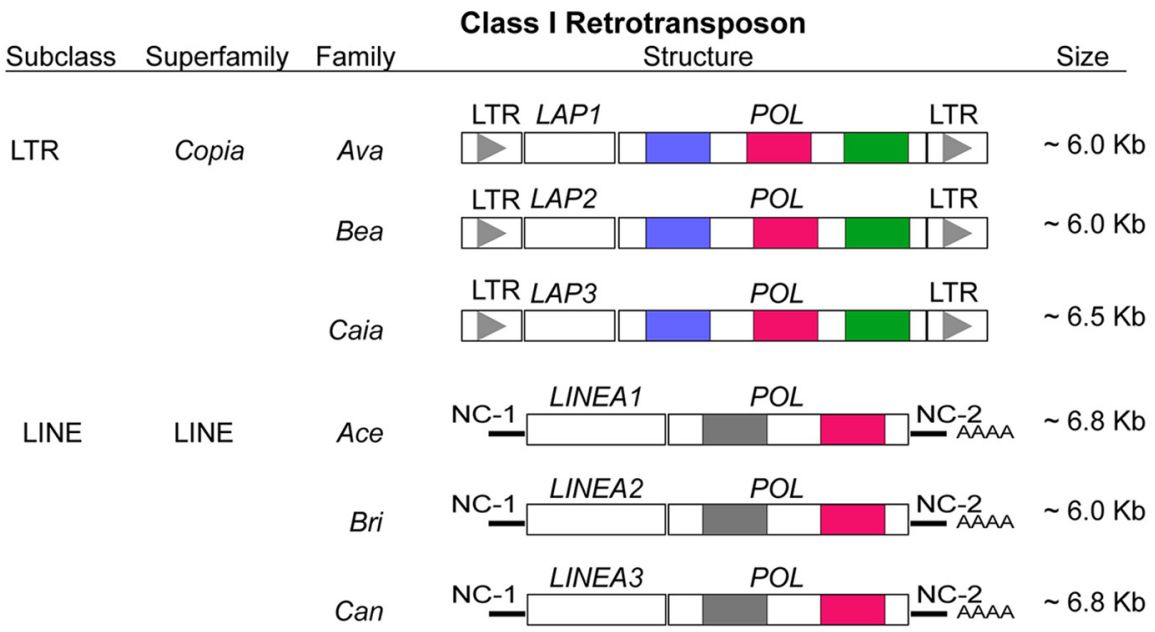

Key:

Endonucleases

B

\section{DNA Transposons}

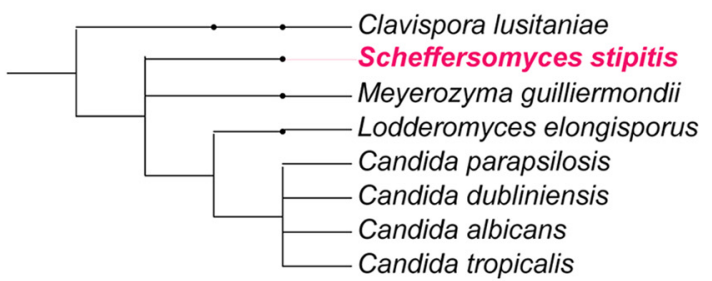

\begin{tabular}{|c|}
\hline $\mathrm{X}$ \\
\hline $\mathrm{X}$ \\
\hline $\mathrm{X}$ \\
\hline $\mathrm{X}$ \\
\hline $\mathrm{X}$ \\
\hline $\mathrm{V}$ \\
\hline $\mathrm{V}$ \\
\hline $\mathrm{V}$ \\
\hline
\end{tabular}

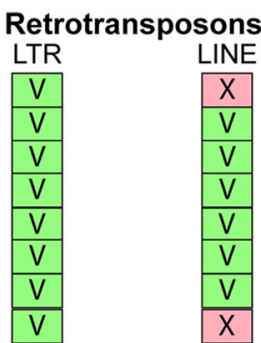

C
Telomeric repeats Clavispora lusitaniae TCTT'TAGGGAGGTAC' - . . - . - . TGATG Scheffersomyces stipitis - - - - ATGGATCTTTTCACGTCTTGCGG Lodderomyces elongisporus - . - . AAGGATGCACT TGAAACTCGGTGT Candida albicans - . - . ACGGATGT - CTAACT TCT TGGTGT Candida tropicalis - - - - AGGGATGT - CACGATCATTGGTGT Saccharomyces cerevisiae $T(G)_{2 \cdot 3}(T G)_{1-6}$

D

Chr1

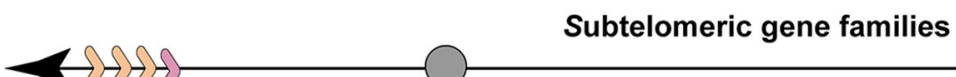

Chr2

Key:

ATP-dependent DNA helicase

$\int$ Fungal-specific Transcription Factor

7) MFS Transporter

$\int$ Agglutinin-like protein

Bea LTR

Ace NC-1

Subtelomeric gene

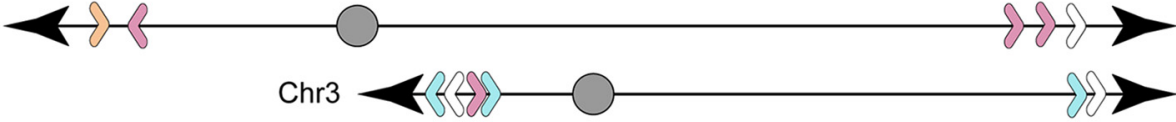

FIG 1 Classification of noncentromeric S. stipitis repeats. (A) Schematics of noncentromeric retrotransposons identified in this study. For each transposon, the subclass, superfamily, and family is indicated. The organization of coding and noncoding sequences of each transposon is 
5/16 subtelomeric regions (Fig. 1D; see also Table S5). No full-length retrotransposons are detected at these genomic locations. Subtelomeric regions contain several gene family members, including gene encoding for ATP-dependent DNA helicases (found in 7/16 subtelomeres), fungus-specific transcription factors (8/16 subtelomeres), MFS transporters (8/16 subtelomeres), and agglutinin-like proteins (11/16 subtelomeres) (Fig. 1D; see also Table S5) (31). In summary, our analysis demonstrates that the $S$. stipitis genome contains several classes of repetitive elements that could be major contributors to genome plasticity.

S. stipitis natural isolates have distinct genomic organizations. Having identified S. stipitis DNA repeats, our next step was to examine S. stipitis phenotypic and genotypic diversity across a geographically diverse set of strains $(n=27)$ that were collected in different habitats (see Table S1, source NRRL and NCYC collection) and that include the sequenced Y11545 strain (31). rDNA fingerprinting confirm that all isolates belong to the $S$. stipitis species (D1/D2 domain of the 26S rDNA similarity $>99 \%$ ) (see Table S6). Phenotypic analyses established that the natural isolates vary in their ability to utilize and grow on different carbon sources. Indeed, compared to the reference Y-11545 strain, different natural isolates cultured in synthetic complete media containing the hexose sugar glucose (SC-G), the pentose sugar xylose (SC-X), or a mixture of both sugars as found in lignocellulose (SC-G+X) display a distinct growth rate, maximum culture density, and lag phase (Fig. 2A; see also Table S7). To determine whether the natural isolates have distinct genomic organizations, we analyzed their karyotype by chromosomes Contour-clamped Homogenous Electric Field (CHEF) gel electrophoresis, a technique allowing chromosome separation according to size. The CHEF electrophoresis analysis reveals clear differences in chromosome patterns demonstrating that S. stipitis natural isolates have a genome organized in different-sized chromosomes (Fig. 2B). We concluded that intraspecies phenotypic and genotypic variation is a common feature of S. stipitis.

Hybrid genomic sequencing identifies transposable elements as drivers of $\boldsymbol{S}$. stipitis genome plasticity. To date, only one S. stipitis isolate (Y-11545) has been sequenced and assembled at chromosome level (31). To gain insights into S. stipitis genetic diversity, we generated a chromosome-level sequence assembly of a second $S$. stipitis natural isolate (Y-7124) by combining MinION Nanopore with Illumina genome sequencing. This isolate was chosen because (i) karyotypic analysis reveals that its genomic organization is distinct from the genomic organization of the reference strain Y-11545 and (ii) Y-7124 is widely used both for industrial applications and for basic research (36).

The Y-7124 genome was sequenced to $186.88 \times$ coverage resulting in a $15.69-\mathrm{Mb}$ assembly arranged in 10 contigs (see Table S8). High-accuracy reads from Illumina sequencing enabled the correction of errors that are associated with the MinION technology. A final chromosome-level assembly was produced by manually identifying overlapping regions between contigs. Comparing the Y-7124 and Y-11545 nucleotide sequences reveals that the two natural isolates overall share a similar coding DNA sequence. The total number of single-nucleotide polymorphisms (SNPs) between the two natural isolates is 50,495, equating to one variant every 306 bases. The majority of these SNPs are synonymous changes $(16,294=74.25 \%)$, while $\sim 25 \%(5,622)$ of the SNPs are missense, and only $0.13 \%$ (28) are nonsense (see Table S9). Despite this high DNA sequence similarity, the Y-7124 genome is organized in eight chromosomes with different sizes and organizations from that of $\mathrm{Y}-11545$ (Fig. 3A). Comparison of the $\mathrm{Y}$ 7124 and $Y-11545$ genomes establishes that retrotransposons are significant drivers of S. stipitis genome diversity as one of the most prominent differences between the two genomes is the abundance and localization of these retrotransposons (Fig. 3B). Indeed, the number of LTR and LINE noncentromeric retrotransposons and transposon-derived

FIG 1 Legend (Continued)

displayed. (B) Cladogram showing CTG(Ser1) clade species with known transposable elements (this study; see also references 18 and 34$)$. The presence $(V)$ or absence $(X)$ of a TE is indicated. (C) Sequence alignment of telomeric terminal repeats in members of the CTG(Ser 1$)$ clade (C. lusitaniae, S. stipitis, L. elongisporus, C. albicans, and C. tropicalis) (this study; see also references 18 and 34). Consensus sequence to the S. cerevisiae telomeric repeats is indicated (magenta box). (D) Schematics of gene family members associated with $\mathrm{S}$. stipitis subtelomeres (30 kb from chromosome end). 
A

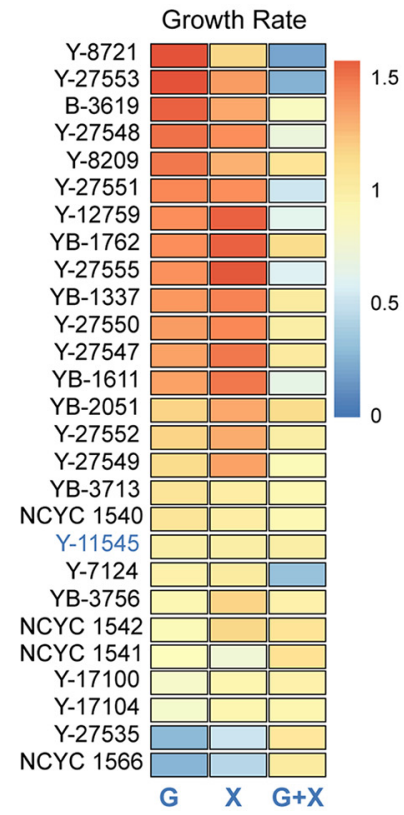

$\operatorname{Max} \mathrm{OD}_{600}$

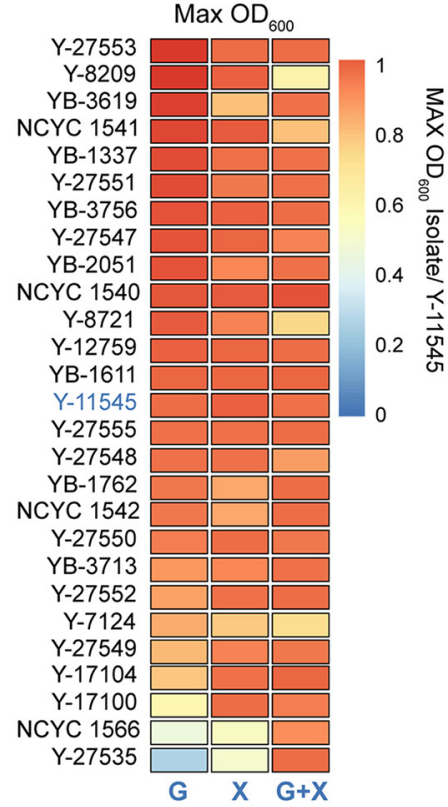

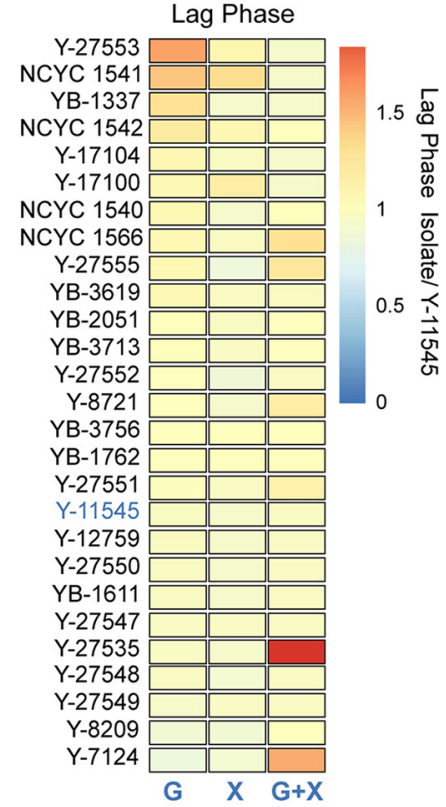

B
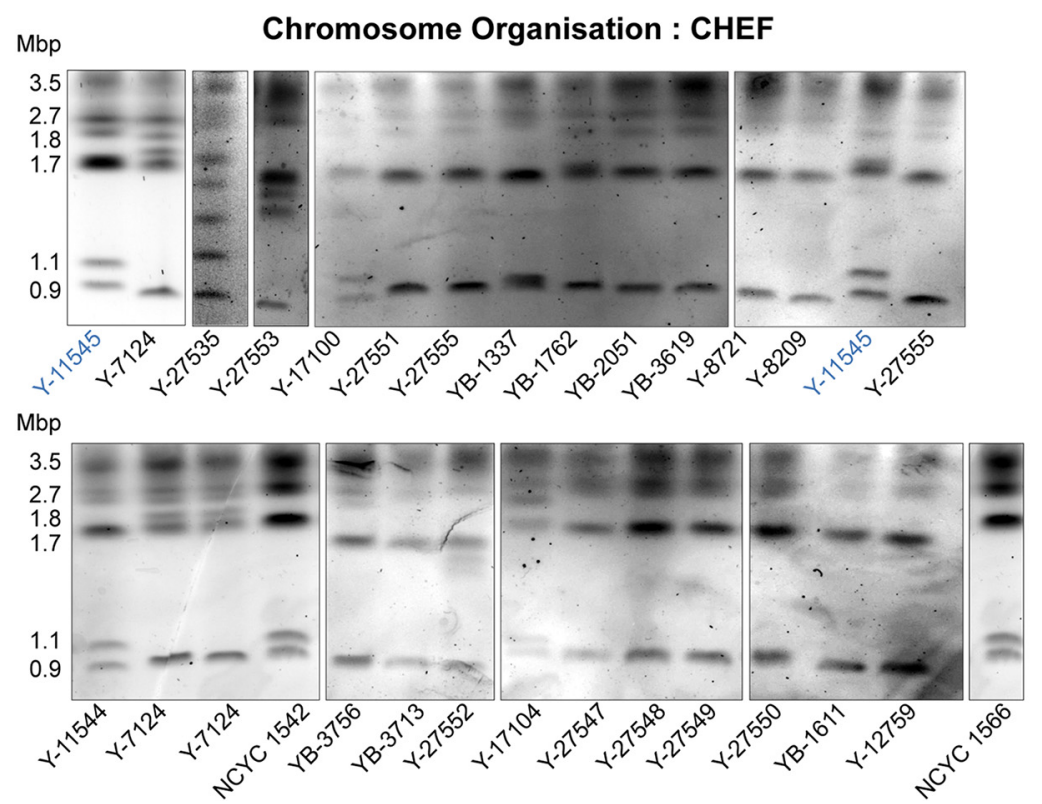

FIG 2 Phenotypic and genotypic diversity in S. stipitis. (A) Heatmaps comparing growth rate (left), maximum OD (middle), and lag time (right) for each S. stipitis natural isolate in comparison to the reference Y-11545 strain (blue). Analyses were performed in glucose $(G)$, xylose $(X)$, and glucose/xylose $(G+X)$ media. The heatmap data are the average of three biological replicates. (B) Karyotyping of S. stipitis natural isolates by CHEF electrophoresis. The Y-11545 strain is highlighted in blue, and the sizes of its eight chromosomes are indicated.

repeats is greater in the $\mathrm{Y}-11545$ reference genome compared to the $\mathrm{Y}-7124$ genome: retrotransposons, solo LTRs, and truncated LINE elements account for approximately $2 \%$ of the reference Y-11545 genome and only for $\sim 1 \%$ of the $\mathrm{Y}-7124$ genome (Fig. 3C). We classified retrotransposons loci present in both isolates (ancestral loci), those present in the reference Y-11545 genome but absent in Y-7124 (deletion loci), and those not present in the reference genome but present in a given strain (insertion loci). Of 69 transposon loci, only 10 ancestral loci ( 15\%) were detected in the two isolates. These sites are likely to be inactive transposons or transposons that rarely transpose. In addition, we detected 42 deletion loci (60\%) and 17 (24\%) insertion loci 
A

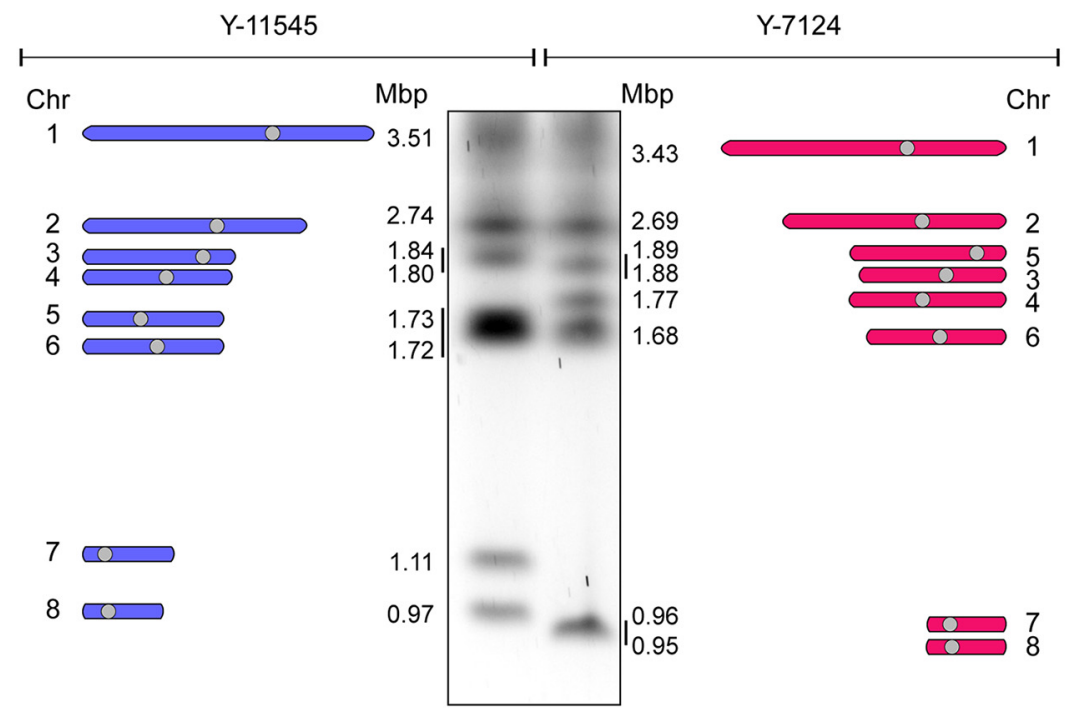

B

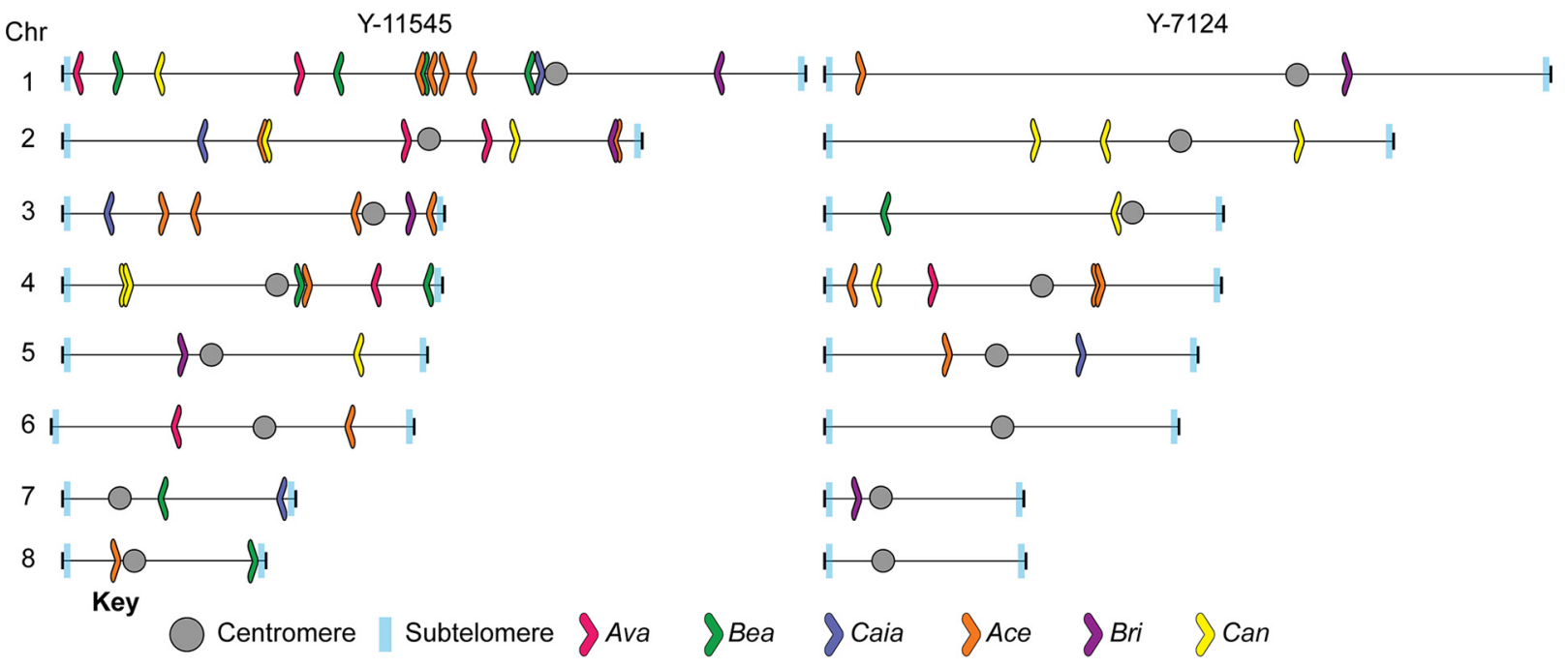

C
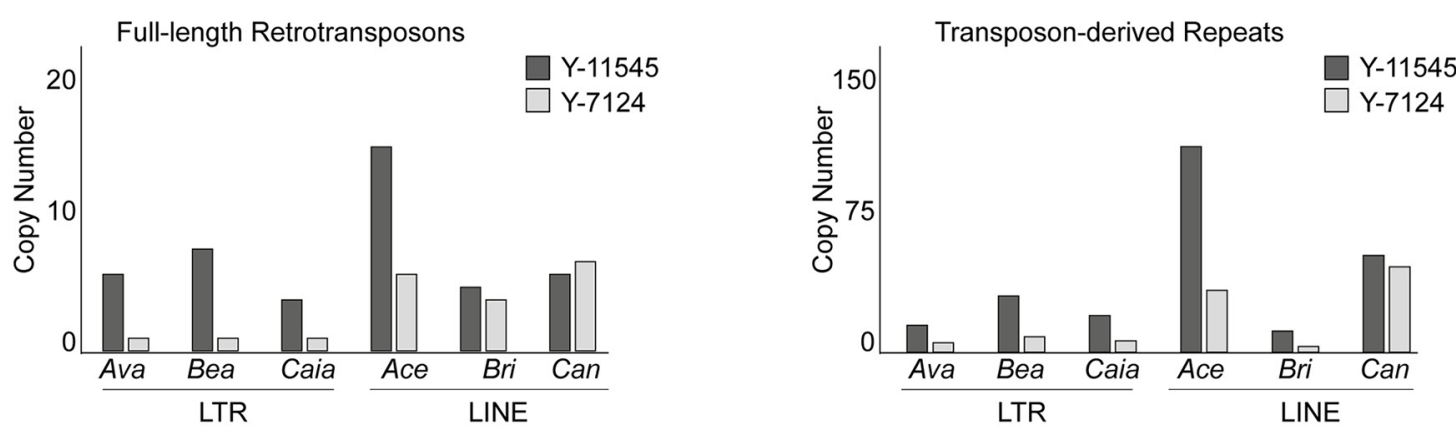

D

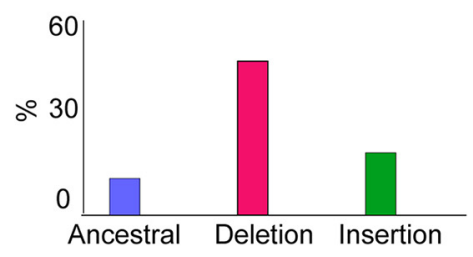

FIG 3 Differences in TE distribution and organization. (A) The genomic organization of Y-11545 and Y-7124 is distinct. (Left) Schematics of Y11545 chromosome organization. The chromosome (Chr) number and size (Mbp) are indicated. (Middle) Karyotyping of S. stipitis Y-11545 and

(Continued on next page) 
(Fig. 3D). The presence of deletion and insertion loci suggests that S. stipitis LTR transposons and LINE elements are active and competent for transposition. Although active transposons can insert into genes to cause functional consequences (37), we did not detect any TE-driven alteration in coding regions.

Transposable elements are sites of chromosome rearrangements. Comparison of the Y-11545 and Y-7124 genome reveals that transposon-rich regions are sites of complex chromosome rearrangements since a transposon-rich region is the breakpoint of a reciprocal translocation between chromosome 5 and chromosome 7 . Indeed, chromosome $5^{\mathrm{Y}-7124}$ (Chr5 ${ }^{\mathrm{Y}-7124}$ ) comprises two parts: one corresponding to $\mathrm{Chr}^{\mathrm{Y}-11545}$ (pink) and one corresponding to $\mathrm{Chr} 5^{\mathrm{Y}-11545}$ (gray). Likewise, $\mathrm{Chr} 7^{\mathrm{Y}-7124}$ contains sequences corresponding to $\mathrm{Chr} 5^{\mathrm{Y}-11545}$ (purple) and one corresponding to $\mathrm{Chr}^{\mathrm{\gamma}-11545}$ (gray) (Fig. 4A and B).

This translocation causes the size change of $\mathrm{Chr}^{\mathrm{Y}-7124}$ and $\mathrm{Chr}^{\mathrm{Y}-7124}$ detected by CHEF karyotyping (Fig. 4A). Southern analyses with a probe specific for Chr5 $5^{\mathrm{Y}-11545}$ (nt 448855 to 449034) confirms that this genomic sequence is associated with the smaller chromosome of $\sim 0.96 \mathrm{Mbp}$ (Chr7 size) in strain Y-7124 (see Fig. S1). The evolutionary history of Y-11545 and Y-7124 is unknown, and therefore it is difficult to predict the molecular events underlying these genomic changes. However, sequence analysis of the rearrangement breakpoint reveals that this structural variation occurs in a genomic region that (i) contains homologous sequences between chromosomes 5 and 7 and (ii) is transposon-rich and contains two inverted repeats on chromosome 7 (Fig. 4B). A second significant difference between the genome organization of $\mathrm{Y}-11545$ and $\mathrm{Y}-7124$ is found at subtelomeric regions: these regions differ in the number and organization of subtelomeric gene families and in the number of transposon-associated repeats (Fig. 4C). Lastly, we detected a distinct centromeres organization where the numbers of Tps5 retrotransposons, LTRs and LARD regions differ between the two isolates (Fig. 4D). The presence of transposons and transposon-derived repeats associated with all these genomic locations strongly suggest that retrotransposons have mediated the chromosomal rearrangement by recombination-mediated mechanisms. Therefore, changes in transposons organization are responsible for the bulk of genomic changes identified in two different natural isolates.

S. stipitis real-time evolution leads to extensive genomic changes. Our results demonstrate that intraspecies genetic diversity is common in S. stipitis. However, since the evolutionary history of the analyzed natural isolates is unknown, it is difficult to predict whether the observed genomic changes are due to the selection of rare genomic rearrangements events. To determine the time scale of $S$. stipitis genome evolution, we investigated the genome organization of 72 single colonies passaged daily for 8 weeks (56 passages, $\sim 672$ divisions) in SC-G $+X$, since its sugar composition resembles what found in lignocellulose (29) (Fig. 5A). Strains were grown at $30^{\circ} \mathrm{C}$, a temperature that does not lead to any growth defect, and $37^{\circ} \mathrm{C}$, a stressful temperature that strongly inhibits S. stipits growth (Fig. 5B). CHEF gel electrophoresis was conducted to identify possible changes in the chromosome organization of the evolved strains. This analysis identifies genome rearrangements in 19/36 strains evolved at $30^{\circ} \mathrm{C}$ and $12 / 36$ strains evolved at $37^{\circ} \mathrm{C}$ (blue and magenta, Fig. 5C). Thus, changes in chromosome organization were detected in the presence $\left(37^{\circ} \mathrm{C}\right)$ or absence $\left(30^{\circ} \mathrm{C}\right)$ of stress. To test whether chromosome rearrangements are associated with a fitness benefit, we tested the ability of the parental, $30^{\circ} \mathrm{C}$-evolved, and $37^{\circ} \mathrm{C}$-evolved strains to grow in SC-G+X media at permissive $\left(30^{\circ} \mathrm{C}\right)$ and restrictive $\left(37^{\circ} \mathrm{C}\right)$ temperatures (Fig. 5D). This analysis demonstrates that $30^{\circ} \mathrm{C}$-evolved strains, with or without chromosomal rearrangements, do not grow well at the restrictive temperature (Fig. 5D). Similarly, $37^{\circ} \mathrm{C}$-evolved strains with no chromosomal rearrangement grow poorly at $37^{\circ} \mathrm{C}$ (Fig. 5D). In contrast, $5 / 1237^{\circ} \mathrm{C}$-evolved strains with chromosome rearrangements grow better than the parental strain at this restrictive temperature (Fig. 5D). This result suggests that changes in chromosome organization have an adaptive

FIG 3 Legend (Continued)

Y-7124 strains by CHEF electrophoresis. (Right) Schematics of Y-7124 chromosome organization. The chromosome (Chr) number and size $(\mathrm{Mbp})$ are indicated. (B) Schematics of noncentromeric transposon family distribution in Y-11545 (left) and Y-7124 (right). (C) Copy numbers of full-length transposons (Left) and transposon-associated repeats associated with the Y-11545 (dark gray) and Y-7124 (light gray) genome. (D) Percentages (\%) of ancestral, deletion, and insertion sites associated with the Y-11545 and Y-7124 genomes. 
A

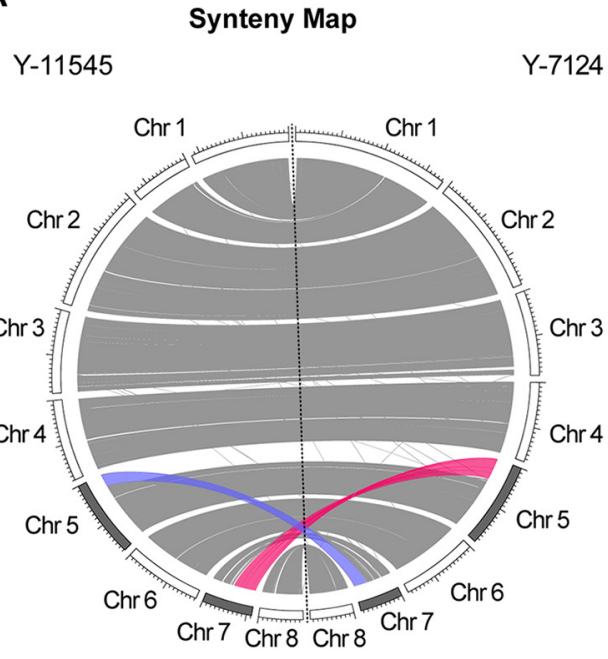

B

Y-11545

Y-7124
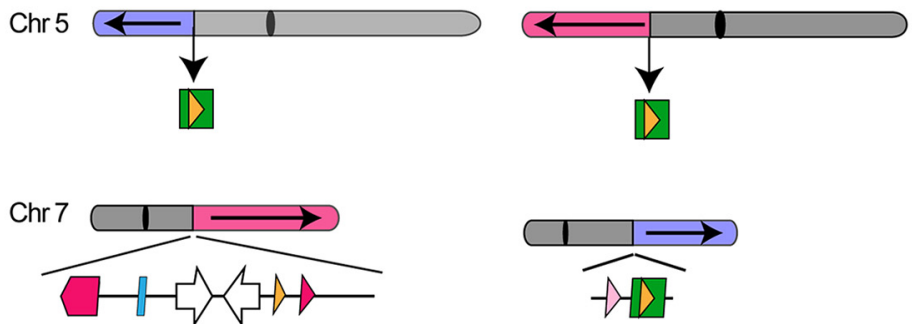

Repeats

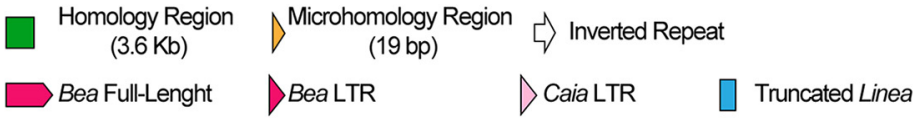

C

Subtelomeric Gene Families and partial TEs

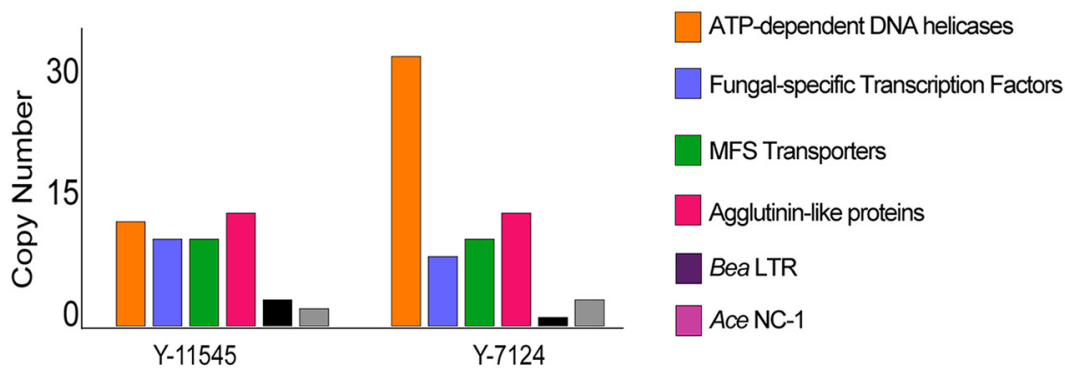

D

Centromeres

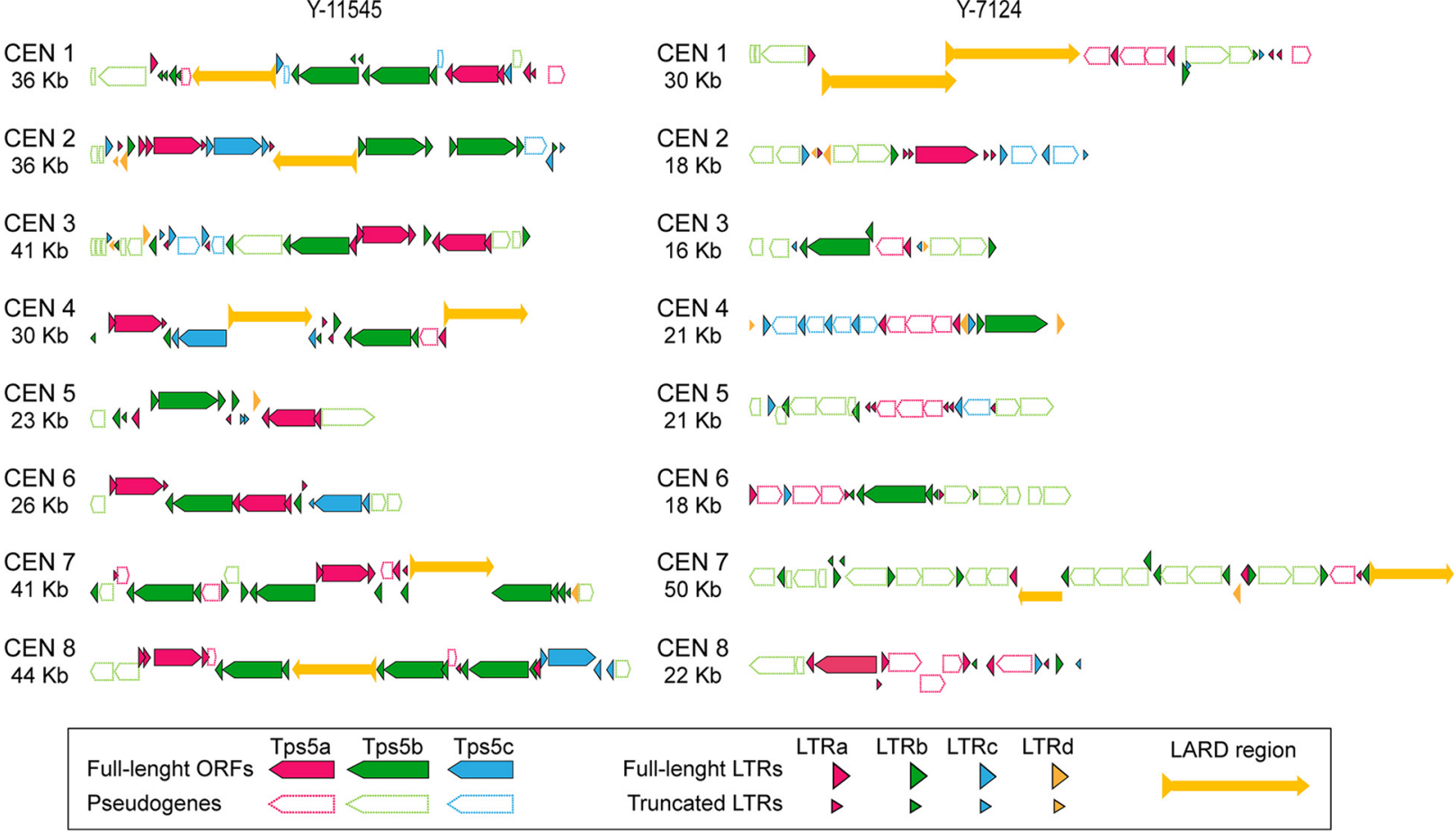

FIG 4 Chromosome rearrangements between S. stipitis natural isolates. (A) Circos plot displaying macrosynteny between Y-11545 (left) and Y7124 (right). Chromosome (Chr) number and size is indicated. Reciprocal translocation between the two genomes is highlighted in purple and

(Continued on next page) 
value. Thus, genome plasticity is a defining feature of the S. stipitis genome, and its genome can rapidly change in mitotic cells propagated in vitro. Our results strongly suggest that the extensive genomic changes can lead to adaptation to hostile environments.

\section{DISCUSSION}

We demonstrate here that the yeast $S$. stipitis has a plastic genome and that genome plasticity is linked to adaptation to hostile environments. We show that noncentromeric retrotransposons are significant drivers of S. stipitis genome diversity. These findings have important implications for developing economically viable second-generation biofuels and better understanding the CTG(Ser1) clade biology.

Retrotransposon are drivers of $\mathbf{S}$. stipitis genome diversity. Our repetitive sequence analysis demonstrates that $S$. stipitis has a DNA repeat content typical of the CTG(Ser1) clade, including TEs, noncanonical terminal telomeric repeats, and subtelomeric regions. As observed in other members of the $\mathrm{CTG}(\operatorname{Ser} 1)$ clade (18), we did not detect any DNA transposons or MRS.

One of our major findings is that that noncentromeric retrotransposons are significant drivers of S. stipitis genome diversity. Our data support the hypothesis that S. stipitis TEs generate genome diversity via two distinct mechanisms: transposition into new genomic locations and recombination-mediated chromosome rearrangements. Indeed, we demonstrated that the number and genomic position of noncentromeric retrotransposons vary between the Y-11545 and Y-7124 S. stipitis isolates. Significantly, we did not detect transposon insertions into coding regions. However, transposons might alter $S$. stipitis gene expression by inserting into gene regulatory regions (37). We propose that $S$. stipitis transposons are active and generate genome diversity by jumping into different genomic locations. Our data also indicate that TEs can generate further genome diversity through either homologous recombination of nearly identical TE copies or by faulty repair of double-strand breaks generated during transposable elements excision (37). Indeed, we find that the translocation breakpoint between chromosome 5 and chromosome 7 is enriched in retrotransposons. Furthermore, TErich subtelomeric regions and centromeres have a distinct organization in the two analyzed isolates, suggesting that the transposons drive this genetic diversity. We hypothesize that transposons elements cause the genetic variability observed during laboratory passaging. In the future, it will be important to combine the hybrid genome sequencing approaches and RNA sequencing to identify the underlying genomic and transcriptional changes.

Genome plasticity and production of second-generation biofuels. One of our key findings is that the $S$. stipitis genome is intrinsically plastic and that chromosome rearrangements are frequent events under stress or unstressed conditions. Secondgeneration biofuels, generated by fermentation of agriculture and forestry waste, have an enormous potential to meet future energy demands and significantly reduce petroleum consumption. To meet the requirements for industrial applications, second-generation biofuels need to be generated by microorganisms that can efficiently utilize and ferment all the sugars found in lignobiomass (38). Consequently, S. stipitis is one of the most promising yeasts for producing second-generation bioethanol since it can efficiently ferment both hexose and pentose sugars $(25,26,29)$. However, robust economically viable $S$. stipitis platforms still require significant development as this organism struggles to survive under the harsh environments generated during second-generation biofuel production. For example, S. stipitis growth and fermentation is inhibited by the chemical pretreatment required to extract glucose and xylose from lignobiomass (36). Growth is also inhibited at high ethanol concentrations, and S. stipitis ferments xylose less efficiently than glucose. Evolutionary engineering approaches under

FIG 4 Legend (Continued)

pink. (B) Schematics of repetitive sequences associated with the translocation junction in the Y-11545 (left) and Y-7124 (right) genomes. (C) Subtelomeric gene families and TEs distribution in the Y-11545 and Y-7124 genomes. (D) Schematics of centromere organization in the Y-11545 (left) and Y-7124 (right). 
A

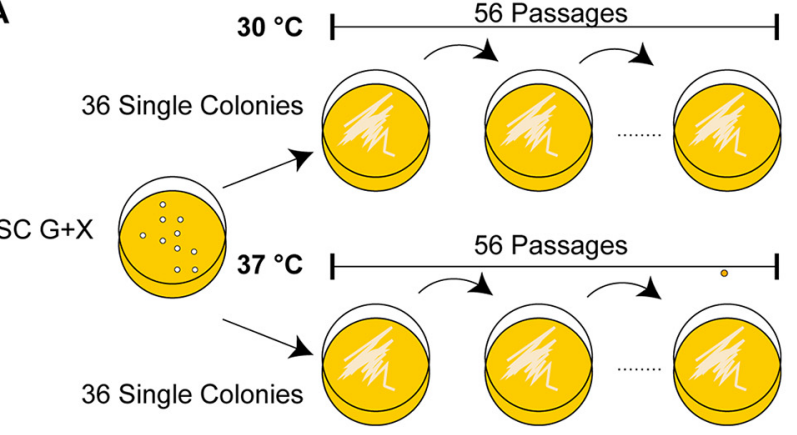

B

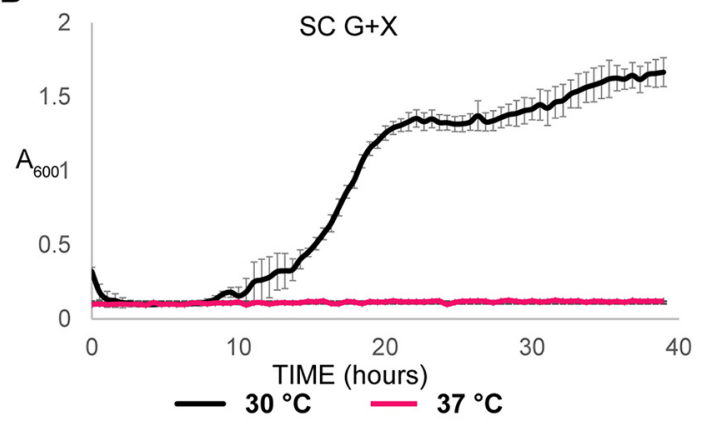

C

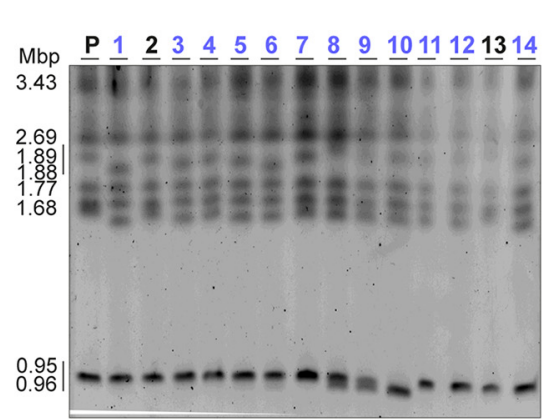

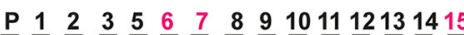

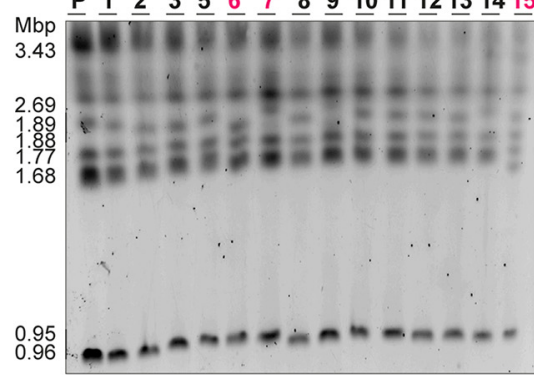

D

$30{ }^{\circ} \mathrm{C}$ Evolution

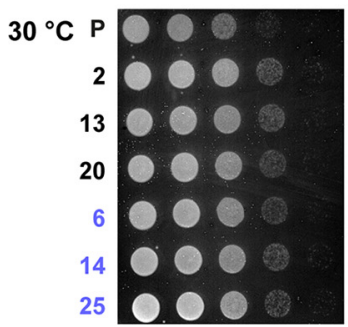

$37^{\circ} \mathrm{C}$

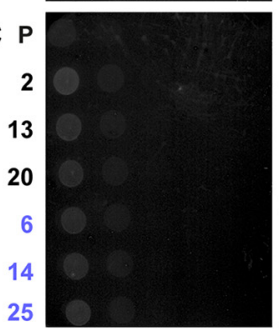

NO

NO $30^{\circ} \mathrm{C}$

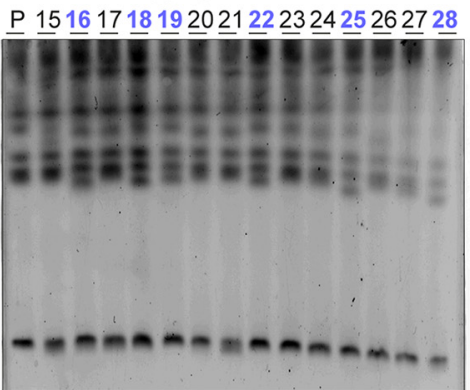

$37^{\circ} \mathrm{C}$
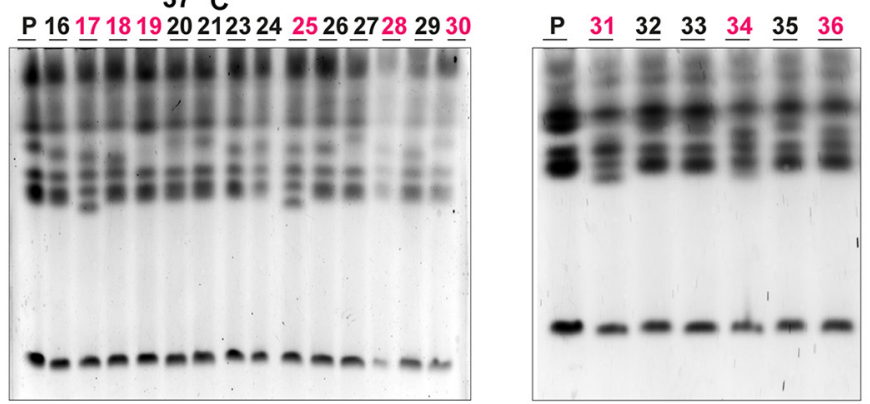

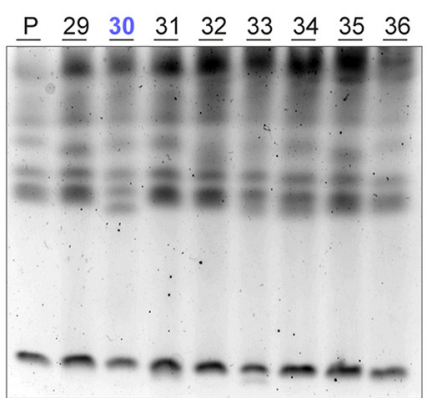

$37^{\circ} \mathrm{C}$ Evolution

NO Rearrangements $30{ }^{\circ} \mathrm{C} \quad \mathrm{P} O \mathrm{O} . \mathrm{O}$. 200.0 \& Rearrangements Rearrangements

Rearrangements Rearrangements
21000 영 20

32000 紋 ..

$33 \bigcirc \bigcirc \bigcirc 96$

$35000 \div$

$27 \mathrm{OOO} \%$

$37^{\circ} \mathrm{C}$

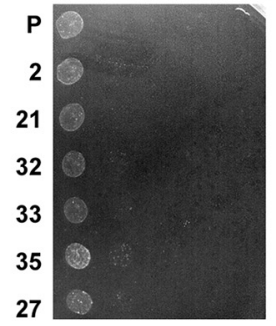

Chromosome Rearrangements $\mathrm{POOO}: \mathrm{POOO}$ : $6 \dot{0} 0$ \%

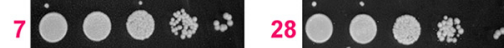
$15 \bigcirc \bigcirc 0 \% 30000$ 영 190.00 \% $\because{ }_{36} \mathrm{O} \bigcirc \mathrm{O} \because$

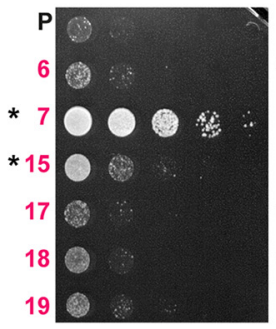

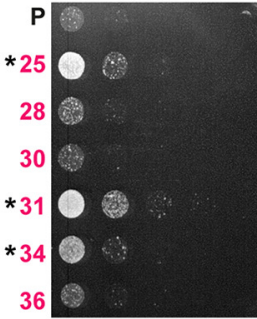
17000 : $3100 \%$. $18000 \% 34000$ :

FIG 5 In vitro evolution induces S. stipitis genome plasticity. (A) Schematics of laboratory evolution strategy. (B) S. stipitis growth curve in $\mathrm{SC} \mathrm{G}+\mathrm{X}$ liquid media at permissive $\left(30^{\circ} \mathrm{C}\right)$ and restrictive $\left(37^{\circ} \mathrm{C}\right)$ temperature. (C) Karyotype organization of $\mathrm{S}$. stipitis colonies after 
selective conditions (i.e., the presence of inhibitory compounds, high concentrations of xylose or ethanol) have been applied to isolate better-performing S. stipitis strains (36).

Our data predict that the genetic makeup and associated improved phenotypes of superior biofuel producer strains are unstable and that the genetic drivers of improved phenotypes might be lost over time. This hypothesis could explain why short-read Illumina genome sequencing has failed to identify point mutations or indels that could explain the superior performance of S. stipitis strains (39). It is also possible that S. stipitis superior strains carry stable complex chromosomal rearrangements with a breakpoint at DNA repeats. Such rearrangements could not have been identified by Illumina sequencing since short-sequenced fragments will not resolve changes associated with long repetitive elements. Thus, economically viable use of S. stipitis for second-generation biofuels production will require an in-depth analysis of the genomic structures of superior strains.

Genome plasticity in the CTG(Ser1) clade. The CTG(Ser1) clade is an incredibly diverse yeast group that includes many important human pathogens and nonpathogenic species (17). Our data support the hypothesis that genome plasticity is a general feature of the CTG(Ser1) yeast clade as it has been observed in C. albicans and S. stipitis $(21,22,40$; this study), two organisms with very different lifestyle. Indeed, while C. albicans is a diploid opportunistic human fungal pathogen that lives almost exclusively in the human host; S. stipitis is a haploid nonpathogenic yeast found in the gut of woodingesting beetles hardwood forests or areas high in agricultural waste $(29,41)$. Furthermore, while C. albicans lacks a canonical sexual cycle and its associated meiosis, S. stipitis has a canonical sexual cycle whereby mating of haploid cells generate diploid cells that undergo meiosis and produce haploid spores (30).

Our results highlight that stress might regulate genome plasticity differently in $C$. albicans and S. stipitis. It has been demonstrated that stress exacerbates $C$. albicans genome instability $(21,42)$. In contrast, results presented in this study suggest that S. stipitis genome instability is not regulated by stress since we detected a similar rate of chromosomal rearrangements when cells are continuously passaged in unstress $\left(30^{\circ} \mathrm{C}\right)$ or stress $\left(37^{\circ} \mathrm{C}\right)$ conditions. Importantly, we also demonstrated that the large genomic changes are associated with fitness benefits suggesting that genome plasticity is instrumental for adaptation to hostile environments.

In summary, our study demonstrates for the first time that S. stipitis genome is plastic. Understanding the cause and effect of this extensive genome plasticity is paramount to understanding the biology of the CTG(Ser1) clade of fungi.

\section{MATERIALS AND METHODS}

Yeast strains and growth conditions. Strains were obtained from the Agricultural Research Service (ARS) Collection, run by the Northern Regional Research Laboratory (NRRL; Peoria, IL), or the National Collection of Yeast Cultures (NCYC; Norwich, United Kingdom) (see Table S1) and confirmed by sequencing (primers AB798 and AB799 of the 26S rDNA [D1/D2 domain]) (43) (see Table S2). Routine culturing was performed at $30^{\circ} \mathrm{C}$ with $200-r p m$ agitation on yeast extract/peptone/D-glucose (YPD) media. Phenotypic and in vitro evolution analyses were conducted on synthetic complete (SC) media containing glucose (SC-G), xylose (SC-X), or a mixture of $60 \%$ glucose and $40 \%$ xylose (SC-G+X). SC-G was used as a reference medium since glucose is the preferred carbon source for both the model system S. cerevisiae and S. stipitis, SC-X was used because of $S$. stipitis unique ability to utilize xylose as a carbon source, and SC-G+X was used because this sugar combination resembles the ratio found in lignocellulose (28). Uridine ( $0.08 \mathrm{~g} /$ liter in YPD and SC) and adenine hemisulfate ( $0.05 \mathrm{~g} /$ liter in YPD) were added as growth supplements. Solid media were prepared by adding $2 \%$ agar.

CHEF electrophoresis. Intact yeast chromosomal DNA was prepared as previously described (44). Briefly, cells were grown overnight, and spheroplasts were prepared in an agarose plug by treating cells $\left(\mathrm{OD}_{600}=\sim 7\right)$ with $0.6 \mathrm{mg} / \mathrm{ml}$ Zymolyase $100 \mathrm{~T}$ (Amsbio, catalog no. 120493-1) in 1\% Low-Melt agarose

FIG 5 Legend (Continued)

8 weeks of laboratory evolution at 30 and $37^{\circ} \mathrm{C}$. Gross chromosomal rearrangements following laboratory evolution at $30^{\circ} \mathrm{C}$ (blue) and $37^{\circ} \mathrm{C}$ (magenta) are indicated. The low resolution of $\mathrm{CHEF}$ detects changes in smaller chromosomes more precisely than changes in larger chromosomes. (D) Serial dilution assay showing growth at 30 and $37^{\circ} \mathrm{C}$ of the following strains: parental $(\mathrm{P}), 30^{\circ} \mathrm{C}$-evolved strains without (NO Rearrangements) or with (Rearrangements) and $37^{\circ} \mathrm{C}$-evolved strains without (NO Rearrangements) or with (Rearrangements). The CHEF analysis strain number is indicated. ${ }^{*}$, Colonies with a fitness advantage compared to the parental strain. 
(Bio-Rad, catalog no. 1613112). Chromosomes were separated in a 1\% Megabase agarose gel (Bio-Rad) in $0.5 \times$ TBE using a CHEF DRII apparatus. Run conditions were as follows: 60 - to 120 -s switch at $6 \mathrm{~V} / \mathrm{cm}$ for $12 \mathrm{~h}$, followed by a 120 - to $300-\mathrm{s}$ switch at $4.5 \mathrm{~V} / \mathrm{cm}$ for $12 \mathrm{~h}$ at $14^{\circ} \mathrm{C}$. Chromosomes were visualized by staining the $0.5 \times$ TBE gel with ethidium bromide $(0.5 \mu \mathrm{g} / \mathrm{ml})$ for $30 \mathrm{~min}$, followed by destaining in water for $30 \mathrm{~min}$. Images were capture using a Syngene GBox Chemi XX6 gel imaging system.

Southern blotting. DNA from CHEF gels were transferred overnight to a Zeta-Probe GT Membrane (Bio-Rad, catalog no. 162-0196) in 20 $\times$ SSC and cross-linked using UV (150 mJ). Probing and detection of the DNA were conducted as previously described (45). Briefly, probes were generated by PCR incorporation of DIG-11-dUTP into target sequences according to the manufacturer's instructions (Roche). Chromosome 5-to-chromosome 7 translocation was detected using a probe generated with the primers AB1028 and AB1029. The probe hybridized to two different chromosomes: in strain Y-11545, the probe hybridized with Chr5 (nt 448855 to 449034), and in strain Y-7124, the probe hybridized with $\mathrm{Chr} 7$ (nt 494698 to 494877 ) (see Table S2). The membrane was incubated with the probe overnight at $42^{\circ} \mathrm{C}$ with DIG Easy Hyb (Roche, catalog no. 11603558001). The DNA was detected with anti-digoxigenin-alkaline phosphatase antibody (Roche, catalog no. 11093274910) and CDP Star Ready-to-Use (Roche, catalog no. 12041677001) according to the manufacturers' instructions.

Phenotypic characterization. Growth analyses were performed using a plate reader (Spectrostar Nano; BMG Labtech) in a 96-well plate format at $30^{\circ} \mathrm{C}$ for $48 \mathrm{~h}$ in SC-G, SC-X, or SC-G+X. The growth rate $\left(\mu, \mathrm{h}^{-1}\right)$ was calculated using: $\mu=\left[\ln \left(X_{2}\right)-\ln \left(X_{1}\right)\right] /\left(t_{2}-t_{1}\right)$, where $X_{1}$ is the biomass concentration $\left(\mathrm{OD}_{600}\right)$ at time point $1\left(t_{1}\right.$, lag time), and $X_{2}$ is the biomass concentration $\left(\mathrm{OD}_{600}\right)$ at time point $2\left(t_{2}\right.$, end of exponential growth phase). The maximum OD (OD units) was determined using MAX() from Excel (Microsoft). The lag time (in minutes) was determined visually as the time within which the exponential growth starts. Experiments were performed in three technical and three biological replicates. Heatmaps showing the average of three biological replicates were generated by $\mathrm{R}$ using the library pheatmap. Analysis of variance was performed to study differences in growth rate, maximum OD, and lag time between the strains. The equality of variances presumption was tested using Levene's test, whereas the Shapiro-Wilk test was used to assess the normality of the data. When both assumptions were satisfied, a Tukey's honest significant test was used to determine significant differences between the natural isolates and the reference $\mathrm{Y}-11545$ strain. When the assumption of equal variance was violated, a one-way test was used to indicate significance. In the case of equal variances but a nonnormal distribution of data, the KruskalWallis rank sum test was used to indicate statistical differences, and significance was determined by pairwise testing. A $P$ value lower than 0.05 was considered significant for all these statistical tests. Statistical tests were performed using $\mathrm{R}$.

In vitro laboratory evolution. A single colony of the S. stipitis strain NRRL Y-7124 was grown overnight in $5 \mathrm{ml}$ of YPD at $30^{\circ} \mathrm{C}$, plated in YPD at a cell density of 100 , and grown $48 \mathrm{~h}$ at $30^{\circ} \mathrm{C}$. A total of 36 single colonies were streaked in two SC-G $+\mathrm{X}$ plates and grown at 30 and $37^{\circ} \mathrm{C}$, respectively, and streaked daily for a total of 56 passages (8 weeks). The karyotype variability of the colonies was assessed by CHEF electrophoresis. Phenotypic differences were assessed by spotting assays. Strains with rearrangements were grown overnight in SC-G+X and diluted to an $\mathrm{OD}_{600}$ of 1 . From this, five $1 / 10$ serial dilutions were prepared, and the cells were plated in SC-G+X using a replica plater (Sigma-Aldrich, R2383-1EA) and grown for $48 \mathrm{~h}$ at both 30 and $37^{\circ} \mathrm{C}$. Strains with no karyotypic modifications after evolution were also used as controls.

Identification of DNA repeats. Long sequences (>100 nt) present more than once in the Y-11545 and Y-7124 genomes were identified by aligning each genome to itself using BLASTN. Repetitive elements ( $E<1$ e-04) were manually verified using IGV/SNAPGene, and clustered repeats were combined. This repeat data set was manually examined to further classify it as (i) related to transposable elements, (ii) telomeric repeats, (iii) centromeres, (iv) belonging to protein-coding gene families, and (v) MRS. Transposons were classified using established guidelines (10). Briefly, LTR transposons were identified by detecting two LTR sequences (size, 260 to $430 \mathrm{nt}$ ) flanking an internal coding region. These potential LTR transposons were further annotated for the presence of the following marks: an LTR flanked by a TG and CA dinucleotides, the presence of a primer binding site with homology to S. stipitis tRNAs (GtRNAdb [http://gtrnadb.ucsc.edu/index.html]), the presence of a coding region with homology to pol gene and containing an integrase (INT), reverse transcriptase (RT), and an RNase H (RH) domain. Non-LTR LINE transposons were identified by detecting coding regions homologous to LINE retrotransposons ORF1 (containing a $\mathrm{Zn}$-finger) and ORF2 (containing an endonuclease and a reverse transcriptase domain) and a terminal poly(A) sequence. Retrotransposons were classified into different families based on sequence similarity with a $90 \%$ cutoff. Terminal telomeric tandem repeats were identified using Tandem Repeats Finder (46) with default parameters. Regional centromeres were identified based on them being the only regions of the genome with a large retrotransposon Tps5 cluster (ca. 20 to $40 \mathrm{~kb}$ ) as previously described (33). Gene families were identified by extracting coding regions from our repeat data sets and performing Clustal Omega sequence alignment and PFAMs domain identification using SMART (http:// smart.embl.de) (47). The identified gene families were compared to published information (31). The presence of MRS was explored using BLASTN and by searching for clusters of noncoding tandem repeats, a hallmark of $C$. albicans MRS, with no homology to retrotransposons and not located at chromosome ends. Sequence alignments were visualized with Jalview v2.11.1.0 (48). Phylogenetic trees were generated with phyloT, a phylogenetic tree generator (biobyte.de) using default parameters, and visualized with Itol (https://itol.embl.de/).

Genome sequencing. The genome of S. stipitis isolate Y-7124 was sequenced by Illumina short-read and MinION long-read technologies. To this end, DNA was extracted from an overnight culture using a Qiagen genomic tip 100/G kit (Qiagen, catalog no. 10243) according to manufacturer's protocol. For 
long-read sequencing, MinION (Oxford Nanopore, Oxford, UK) was performed on a DNA library prepared from size-selected gDNA. DNA fragments greater than $30 \mathrm{~kb}$ were selected using a Blue Pippin (Sage Science) and concentrated using AmPure beads. From this, a DNA library was prepared using a 1D ligation sequencing kit (SQK-LSK108) and run on the Oxford Nanopore MinION flow-cell FLOMIN 106D. The same gDNA extract was also used for the preparation of Illumina libraries. In this case, the DNA was sheared using a Covaris M220 with microTUBE-50 (catalog no. 520166) and size selected using the Blue Pippin (Sage Science). The library was constructed using a PCR-free kit with NEBNext End Repair (E6050S), NEBNext dA-tailing (E6053S), and Blunt T/A ligase (M0367S) New England Biolabs modules. Sequencing was performed on a MiSeq benchtop analyzer (Illumina) using a $2 \times 300-b p$ PE (MS-1023003) flow cell.

Genome assembly. Base-calling and demultiplexing were conducted with Albacore v2.3.3 (https:// community.nanoporetech.com). Adapters and low-quality data were trimmed using the eautils package fastq-mcf 1.04.636 (https://expressionanalysis.github.io/ea-utils/). For nanopore sequence data, adapter trimming was performed using Porechop v.0.1.0 (https://github.com/rrwick/Porechop). Genome assembly was completed using long reads, with read correction performed with Canu v1.8 (49), followed by assembly in SMARTdenovo github commit id 61cf13d (50). The draft assembly was corrected using the corrected nanopore reads through five rounds of Racon github commit 24e30a9 (51) and then by raw fast5 files using 10 rounds of Nanopolish v0.9.0 (52). Illumina sequencing reads were then used to polish the resulting assembly through 10 rounds of Pilon v1.17 (53). After genome assembly, BUSCO v3 was run to assess evolutionarily conserved gene content (54), using the Saccharomycetales_odb9 gene database. The Saccharomycetales database contains 1711 genes, which are therefore expected to be present in S. stipitis. Of these, 1,683 (98.36\%) were identified in the Y-7124 assembly, demonstrating a good level of completeness (>95\%) (see Table S8). Assembly size and contiguity statistics were assessed using QUAST v4.5 (55). This initial assembly of the nuclear genome contained 10 contigs. A chromosome-level assembly was produced by identification of overlapping regions between the contigs: a 244-kbp overlapping region between contigs 7 and 2 led to the final assembly of chromosome 1, and an 83-kbp overlapping region between contigs 9 and 10 led to the final assembly of chromosome 8 .

Genome annotation. Genome annotation was performed using FUNGAP v1.0.1 (56) with fastq reads from NCBI SRA accession SRR8420582 (BioSample SAMN09064163) used as RNA-Seq training data and protein sequences taken from NCBI assembly accession GCA_000209165.1 for S. stipitis NRRL Y-11545 (CBS6054) used, for example, proteins. Protein fasta files were extracted from predicted gene models using the yeast mitochondrial code (code 3) and the alternative yeast nuclear code (code 12). Functional annotation of gene models was performed through BLASTp searches versus all proteins from the NCBI reference fungal genomes (downloaded 11 April 2020), retrieving the top-scoring blast hit with an $\mathrm{E}$ value of $<1 \times 10^{-30}$. These annotations were supplemented with domain annotations from Interproscan v5.42-78.0 (57). The annotated genome was submitted to the NCBI, with submission files prepared using GAG v2.0.1 (http://genomeannotation.github.io/GAG.), Annie github commit 4bb3980 (http://genomeannotation.github.io/annie), and table2 as n_GFF v1.23.377 (available from https:/ftp .ncbi.nih.gov/toolbox/ncbi_tools/converters/by_program/tbl2asn/).

Comparative genomics. Whole-genome alignment between Y-7124 and Y-11545 was performed using the nucmer tool from the MUMmer package v4.0 (58), and results were visualized using Circos v0.6 (59). Orthology analysis was performed between predicted proteins from these isolates using OrthoFinder v2.3.11 (60), with results visualized using the package VennDiagram in R (61).

Sequence variants were identified in Y-7124 through comparison to the Y-11545 assembly. Short-read sequence data for Y-7124 were aligned to the reference genome using BWA v0.7.15-r1140 (62) before filtering using using picardtools v2.5.0 to remove optical duplicates (http://broadinstitute.github.io/picard/). SNP and insertion/deletion (InDel) calling were performed using GATK4 (63). Low-confidence variants were then filtered using VCFtools v0.1.15 (64) using minimum mapping quality of 40 , a phred quality of 30 , a read depth of 10 , and a genotype quality of 30. The effect of variants on NRRL Y-11545 gene models was determined using SnpEff v4.2 (65).

Data availability. This Whole Genome Shotgun project has been deposited at DDBJ/ENA/GenBank under the accession JADGGA000000000. The version described here is version JADGGA010000000. Illumina and nanopore sequence data associated with this work have been deposited on the Sequence Read Archive (SRA) under BioProject PRJNA609885.

\section{SUPPLEMENTAL MATERIAL}

Supplemental material is available online only.

FIG S1, TIF file, 1.4 MB.

TABLE S1, DOCX file, $0.02 \mathrm{MB}$.

TABLE S2, DOCX file, $0.01 \mathrm{MB}$.

TABLE S3, XLSX file, $0.02 \mathrm{MB}$.

TABLE S4, XLSX file, $0.01 \mathrm{MB}$.

TABLE S5, XLSX file, $0.02 \mathrm{MB}$.

TABLE S6, DOCX file, $0.01 \mathrm{MB}$.

TABLE S7, DOCX file, $0.02 \mathrm{MB}$.

TABLE S8, DOCX file, $0.01 \mathrm{MB}$.

TABLE S9, DOCX file, $0.01 \mathrm{MB}$. 


\section{ACKNOWLEDGMENTS}

We thank Patricia Slininger, members of the Buscaino Lab, the Kent Fungal Group,

and Jan Soetaert for discussion and critical reading of the manuscript.

This study was supported by the University of Kent Vice-Chancellor's Research Scholarship (to S.V.), BBSRC grants (BB/L008041/1 to A.B., BB/P020364/1 to R.J.H.), and an MRC grant (MR/M019713/1 to A.B.).

\section{REFERENCES}

1. Burns KH. 2017. Transposable elements in cancer. Nat Rev Cancer 17: 415-424. https://doi.org/10.1038/nrc.2017.35.

2. Colnaghi R, Carpenter G, Volker M, O'Driscoll M. 2011. The consequences of structural genomic alterations in humans: genomic disorders, genomic instability and cancer. Semin Cell Dev Biol 22:875-885. https://doi.org/10 .1016/j.semcdb.2011.07.010.

3. Galhardo RS, Hastings PJ, Rosenberg SM. 2007. Mutation as a stress response and the regulation of evolvability. Crit Rev Biochem Mol Biol 42: 399-435. https://doi.org/10.1080/10409230701648502.

4. Buscaino A. 2019. Chromatin-mediated regulation of genome plasticity in human fungal pathogens. Genes (Basel) 10:855. https://doi.org/10.3390/ genes10110855.

5. Hirakawa MP, Martinez DA, Sakthikumar S, Anderson MZ, Berlin A, Gujja S, Zeng Q, Zisson E, Wang JM, Greenberg JM, Berman J, Bennett RJ, Cuomo CA. 2015 Genetic and phenotypic intra-species variation in Candida albicans. Genome Res 25:413-425.

6. Peter J, De Chiara M, Friedrich A, Yue J-X, Pflieger D, Bergström A, Sigwalt A, Barre B, Freel K, Llored A, Cruaud C, Labadie K, Aury J-M, Istace B, Lebrigand K, Barbry P, Engelen S, Lemainque A, Wincker P, Liti G, Schacherer J. 2018. Genome evolution across 1,011 Saccharomyces cerevisiae isolates Species-wide genetic and phenotypic diversity. Nature 556: 339-344. https://doi.org/10.1038/s41586-018-0030-5.

7. Aguilera A, García-Muse T. 2013. Causes of genome instability. Annu Rev Genet 47:1-32. https://doi.org/10.1146/annurev-genet-111212-133232.

8. Fedoroff N. 2000. Transposons and genome evolution in plants. Proc Natl Acad Sci U S A National Academy of Sciences

9. Feschotte C, Pritham EJ. 2007. DNA transposons and the evolution of eukaryotic genomes. Annu Rev Genet 41:331-368. https://doi.org/10.1146/ annurev.genet.40.110405.090448.

10. Wicker T, Sabot F, Hua-Van A, Bennetzen JL, Capy P, Chalhoub B, Flavell A, Leroy $P$, Morgante $M$, Panaud $O$, Paux E, SanMiguel $P$, Schulman AH. 2007. A unified classification system for eukaryotic transposable elements. Nat Rev Genet 8:973-982. https://doi.org/10.1038/nrg2165.

11. Havecker ER, Gao X, Voytas DF. 2004. The diversity of LTR retrotransposons. Genome Biol 5:225. https://doi.org/10.1186/gb-2004-5-6-225.

12. Llorens C, Muñoz-Pomer A, Bernad L, Botella H, Moya A. 2009. Network dynamics of eukaryotic LTR retroelements beyond phylogenetic trees. Biol Direct 4:41-31. https://doi.org/10.1186/1745-6150-4-41.

13. Finnegan DJ. 1997. Transposable elements: how non-LTR retrotransposons do it. Curr Biol 7:R245-R248. https://doi.org/10.1016/S0960-9822(06)00112-6.

14. Januszyk K, Li PWL, Villareal V, Branciforte D, Wu H, Xie Y, Feigon J, Loo JA, Martin SL, Clubb RT. 2007. Identification and solution structure of a highly conserved C-terminal domain within ORF1p required for retrotransposition of long interspersed nuclear element-1. J Biol Chem 282: 24893-24904. https://doi.org/10.1074/jbc.M702023200.

15. Luan DD, Korman MH, Jakubczak JL, Eickbush TH. 1993. Reverse transcription of R2Bm RNA is primed by a nick at the chromosomal target site: a mechanism for non-LTR retrotransposition. Cell 72:595-605. https://doi .org/10.1016/0092-8674(93)90078-5.

16. Papon N, Courdavault V, Clastre M. 2014. Biotechnological potential of the fungal CTG clade species in the synthetic biology era. Trends Biotechnol 32:167-168. https://doi.org/10.1016/j.tibtech.2013.10.006.

17. Gabaldón T, Naranjo-Ortíz MA, Marcet-Houben M. 2016. Evolutionary genomics of yeast pathogens in the Saccharomycotina. FEMS Yeast Res 16:fow064. https://doi.org/10.1093/femsyr/fow064.

18. Butler G, Rasmussen MD, Lin MF, Santos MAS, Sakthikumar S, Munro CA, Rheinbay E, Grabherr M, Forche A, Reedy JL, Agrafioti I, Arnaud MB, Bates S, Brown AJP, Brunke $S$, Costanzo MC, Fitzpatrick DA, De Groot PWJ, Harris D, Hoyer LL, Hube B, Klis FM, Kodira C, Lennard N, Logue ME, Martin R, Neiman AM, Nikolaou E, Quail MA, Quinn J, Santos MC, Schmitzberger FF, Sherlock G, Shah P, Silverstein KAT, Skrzypek MS, Soll D, Staggs R, Stansfield I, Stumpf MPH, Sudbery PE, Srikantha T, Zeng Q,
Berman J, Berriman M, Heitman J, Gow NAR, Lorenz MC, Birren BW, Kellis M, Cuomo CA. 2009. Evolution of pathogenicity and sexual reproduction in eight Candida genomes. Nature 459:657-662. https://doi.org/10.1038/ nature08064.

19. Wohlbach DJ, Kuo A, Sato TK, Potts KM, Salamov AA, LaButti KM, Sun $H$, Clum A, Pangilinan JL, Lindquist EA, Lucas $S$, Lapidus A, Jin M, Gunawan C, Balan V, Dale BE, Jeffries TW, Zinkel R, Barry KW, Grigoriev IV, Gasch AP. 2011. Comparative genomics of xylose-fermenting fungi for enhanced biofuel production. Proc Natl Acad Sci U S A 108:13212-13217. https:// doi.org/10.1073/pnas.1103039108.

20. Krassowski T, Coughlan AY, Shen X-X, Zhou X, Kominek J, Opulente DA, Riley R, Grigoriev IV, Maheshwari N, Shields DC, Kurtzman CP, Hittinger CT, Rokas A, Wolfe KH. 2018. Evolutionary instability of CUG-Leu in the genetic code of budding yeasts. Nat Commun 9:1887. https://doi.org/10 .1038/s41467-018-04374-7.

21. Forche A, Abbey D, Pisithkul T, Weinzierl MA, Ringstrom T, Bruck D, Petersen K, Berman J. 2011. Stress alters rates and types of loss of heterozygosity in Candida albicans. mBio 2:e00129-11. https://doi.org/10.1128/ mBio.00129-11.

22. Todd RT, Selmecki A. 2020. Expandable and reversible copy number amplification drives rapid adaptation to antifungal drugs. Elife 9:1-33. https://doi.org/10.7554/eLife.58349.

23. Todd RT, Wikoff TD, Forche A, Selmecki A. 2019. Genome plasticity in Candida albicans is driven by long repeat sequences. Elife 8:e45954. https:// doi.org/10.7554/eLife.45954.

24. Hirakawa MP, Martinez DA, Sakthikumar S, Anderson MZ, Berlin A, Gujja S, Zeng Q, Zisson E, Wang JM, Greenberg JM, Berman J, Bennett RJ, Cuomo CA. 2015. Genetic and phenotypic intra-species variation in Candida albicans. Genome Res 25:413-425. https://doi.org/10.1101/gr.174623.114.

25. Du Preez JC, van Driessel B, Prior BA. 1989. D-Xylose fermentation by Candida shehatae and pichia stipitis at low dissolved oxygen levels in fed-batch cultures. Biotechnol Lett 11:131-136. https://doi.org/10.1007/BF01192189.

26. Du Preez JC, van Driessel B, Prior BA. 1989. Ethanol tolerance of Pichia stipitis and Candida shehatae strains in fed-batch cultures at controlled low dissolved oxygen levels. Appl Microbiol Biotechnol 30:53-58. https:// doi.org/10.1007/BF00255996.

27. Sims REH, Mabee W, Saddler JN, Taylor M. 2010. An overview of second generation biofuel technologies. Bioresour Technol 101:1570-1580. https://doi.org/10.1016/j.biortech.2009.11.046.

28. Robak K, Balcerek M. 2018. Review of second generation bioethanol production from residual biomass. Food Technol Biotechnol 56:174-187. https://doi.org/10.17113/ftb.56.02.18.5428.

29. Suh SO, Marshall CJ, McHugh JV, Blackwell M. 2003. Wood ingestion by passalid beetles in the presence of xylose-fermenting gut yeasts. Mol Ecol 12:3137-3145. https://doi.org/10.1046/j.1365-294x.2003.01973.x.

30. Melake T, Passoth V, Klinner U. 1996. Characterization of the genetic system of the xylose-fermenting yeast Pichia stipitis. Curr Microbiol 33: 237-242. https://doi.org/10.1007/s002849900106.

31. Jeffries TW, Grigoriev IV, Grimwood J, Laplaza JM, Aerts A, Salamov A, Schmutz J, Lindquist E, Dehal P, Shapiro H, Jin YS, Passoth V, Richardson PM. 2007. Genome sequence of the lignocellulose-bioconverting and xylose-fermenting yeast Pichia stipitis. Nat Biotechnol 25:319-326. https://doi.org/10.1038/nbt1290.

32. Lynch DB, Logue ME, Butler G, Wolfe KH. 2010. Chromosomal G+C content evolution in yeasts: systematic interspecies differences, and GC-poor troughs at centromeres. Genome Biol Evol 2:572-583. https://doi.org/10 .1093/gbe/evq042.

33. Coughlan AY, Wolfe KH. 2019. The reported point centromeres of Scheffersomyces stipitis are retrotransposon long terminal repeats. Yeast 36: 275-283. https://doi.org/10.1002/yea.3375.

34. Jackson AP, Gamble JA, Yeomans T, Moran GP, Saunders D, Harris D, Aslett M, Barrell JF, Butler G, Citiulo F, Coleman DC, de Groot PWJ, 
Goodwin TJ, Quail MA, McQuillan J, Munro CA, Pain A, Poulter RT, Rajandream M-A, Renauld H, Spiering MJ, Tivey A, Gow NAR, Barrell B, Sullivan DJ, Berriman M. 2009. Comparative genomics of the fungal pathogens Candida dubliniensis and Candida albicans. Genome Res 19: 2231-2244. https://doi.org/10.1101/gr.097501.109.

35. Frank AC, Wolfe KH. 2009. Evolutionary capture of viral and plasmid DNA by yeast nuclear Chromosomes. Eukaryot Cell 8:1521-1531. https://doi.org/ 10.1128/EC.00110-09.

36. Slininger PJ, Shea-Andersh MA, Thompson SR, Dien BS, Kurtzman CP, Balan V, da Costa Sousa L, Uppugundla N, Dale BE, Cotta MA. 2015. Evolved strains of Scheffersomyces stipitis achieving high ethanol productivity on acidand base-pretreated biomass hydrolysate at high solids loading. Biotechnol Biofuels 8:1-27. https://doi.org/10.1186/s13068-015-0239-6.

37. Bourque G, Burns KH, Gehring M, Gorbunova V, Seluanov A, Hammell M, Imbeault M, Izsvák Z, Levin HL, Macfarlan TS, Mager DL, Feschotte C. 2018. Ten things you should know about transposable elements. Genome Biol 19:199. https://doi.org/10.1186/s13059-018-1577-z.

38. Jansen MLA, Bracher JM, Papapetridis I, Verhoeven MD, de Bruijn $H$, de Waal PP, van Maris AJA, Klaassen P, Pronk JT. 2017. Saccharomyces cerevisiae strains for second-generation ethanol production: from academic exploration to industrial implementation. FEMS Yeast Res 17:fox044.

39. Smith DR, Quinlan AR, Peckham HE, Makowsky K, Tao W, Woolf B, Shen L, Donahue WF, Tusneem N, Stromberg MP, Stewart DA, Zhang L, Ranade SS, Warner JB, Lee CC, Coleman BE, Zhang Z, McLaughlin SF, Malek JA, Sorenson JM, Blanchard AP, Chapman J, Hillman D, Chen F, Rokhsar DS, McKernan KJ, Jeffries TW, Marth GT, Richardson PM. 2008. Rapid wholegenome mutational profiling using next-generation sequencing technologies. Genome Res 18:1638-1642. https://doi.org/10.1101/gr.077776.108.

40. Selmecki A, Forche A, Berman J. 2010. Genomic plasticity of the human fungal pathogen Candida albicans. Eukaryot Cell 9:991-1008. https://doi .org/10.1128/EC.00060-10.

41. Fisher MC, Hawkins NJ, Sanglard D, Gurr SJ. 2018. Worldwide emergence of resistance to antifungal drugs challenges human health and food security. Science 360:739-742. https://doi.org/10.1126/science.aap7999.

42. Freire-Benéitez V, Gourlay S, Berman J, Buscaino A. 2016. Sir2 regulates stability of repetitive domains differentially in the human fungal pathogen Candida albicans. Nucleic Acids Res 44:9166-9179. https://doi.org/10 .1093/nar/gkw594.

43. Villa-Carvajal M, Querol A, Belloch C. 2006. Identification of species in the genus Pichia by restriction of the internal transcribed spacers (ITS1 and ITS2) and the 5.8S ribosomal DNA gene. Antonie Van Leeuwenhoek 90: 171-181. https://doi.org/10.1007/s10482-006-9071-0.

44. Schwartz DC, Cantor CR. 1984. Separation of yeast chromosome-sized DNAs by pulsed-field gradient gel electrophoresis. Cell 37:67-75. https:// doi.org/10.1016/0092-8674(84)90301-5.

45. Ketel C, Wang HSW, McClellan M, Bouchonville K, Selmecki A, Lahav T, Gerami-Nejad M, Berman J. 2009. Neocentromeres form efficiently at multiple possible loci in Candida albicans. PLoS Genet 5:e1000400. https://doi .org/10.1371/journal.pgen.1000400.

46. Benson G. 1999. Tandem repeats finder: a program to analyze DNA sequences. Nucleic Acids Res 27:573-580. https://doi.org/10.1093/nar/27.2.573.

47. Letunic I, Bork P. 2018. 20 years of the SMART protein domain annotation resource. Nucleic Acids Res 46:D493-D496. https://doi.org/10.1093/nar/gkx922.

48. Waterhouse AM, Procter JB, Martin DMA, Clamp M, Barton GJ. 2009. Jalview version 2: a multiple sequence alignment editor and analysis workbench. Bioinformatics 25:1189-1191. https://doi.org/10.1093/bioinformatics/btp033.

49. Koren S, Walenz BP, Berlin K, Miller JR, Bergman NH, Phillippy AM. 2017. Canu: scalable and accurate long-read assembly via adaptive $\kappa$-mer weighting and repeat separation. Genome Res 27:722-736. https://doi .org/10.1101/gr.215087.116.

50. Liu H, Wu S, Li A, Ruan J. 2021. SMARTdenovo: a de novo assembler using long noisy reads. Gigabyte https://doi.org/10.46471/gigabyte.15.

51. Vaser R, Sović I, Nagarajan N, Šikić M. 2017. Fast and accurate de novo genome assembly from long uncorrected reads. Genome Res 27:737-746. https://doi.org/10.1101/gr.214270.116.

52. Loman NJ, Quick J, Simpson JT. 2015. A complete bacterial genome assembled de novo using only nanopore sequencing data. Nat Methods 12:733-735. https://doi.org/10.1038/nmeth.3444.

53. Walker BJ, Abeel T, Shea T, Priest M, Abouelliel A, Sakthikumar S, Cuomo CA, Zeng Q, Wortman J, Young SK, Earl AM. 2014. Pilon: an integrated tool for comprehensive microbial variant detection and genome assembly improvement. PLoS One 9:e112963. https://doi.org/10.1371/journal.pone.0112963.

54. Simão FA, Waterhouse RM, loannidis $P$, Kriventseva EV, Zdobnov EM. 2015. BUSCO: assessing genome assembly and annotation completeness with single-copy orthologs. Bioinformatics 31:3210-3212. https://doi.org/ 10.1093/bioinformatics/btv351.

55. Gurevich A, Saveliev V, Vyahhi N, Tesler G. 2013. QUAST: Quality assessment tool for genome assemblies. Bioinformatics 29:1072-1075. https:// doi.org/10.1093/bioinformatics/btt086.

56. Min B, Grigoriev IV, Choi I-G. 2017. FunGAP: Fungal Genome Annotation Pipeline using evidence-based gene model evaluation. Bioinformatics 33: 2936-2937. https://doi.org/10.1093/bioinformatics/btx353.

57. Jones $P$, Binns D, Chang HY, Fraser M, Li W, McAnulla C, McWilliam H, Maslen J, Mitchell A, Nuka G, Pesseat S, Quinn AF, Sangrador-Vegas A, Scheremetjew M, Yong SY, Lopez R, Hunter S. 2014. InterProScan 5: genome-scale protein function classification. Bioinformatics 30:1236-1240. https://doi.org/10.1093/bioinformatics/btu031.

58. Marçais G, Delcher AL, Phillippy AM, Coston R, Salzberg SL, Zimin A. 2018. MUMmer4: a fast and versatile genome alignment system. PLoS Comput Biol 14:e1005944. https://doi.org/10.1371/journal.pcbi.1005944.

59. Krzywinski M, Schein J, Birol I, Connors J, Gascoyne R, Horsman D, Jones SJ, Marra MA. 2009. Circos: an information aesthetic for comparative genomics. Genome Res 19:1639-1645. https://doi.org/10.1101/gr.092759.109.

60. Emms DM, Kelly S. 2015. OrthoFinder: solving fundamental biases in whole genome comparisons dramatically improves orthogroup inference accuracy. Genome Biol 16:157. https://doi.org/10.1186/s13059-015-0721-2.

61. Chen H, Boutros PC. 2011. VennDiagram: a package for the generation of highly-customizable Venn and Euler diagrams in R. BMC Bioinformatics 12:35. https://doi.org/10.1186/1471-2105-12-35.

62. Li H, Durbin R. 2009. Fast and accurate short read alignment with Burrows-Wheeler transform. Bioinformatics 25:1754-1760. https://doi.org/10 .1093/bioinformatics/btp324.

63. McKenna A, Hanna M, Banks E, Sivachenko A, Cibulskis K, Kernytsky A, Garimella K, Altshuler D, Gabriel S, Daly M, DePristo MA. 2010. The genome analysis toolkit: a MapReduce framework for analyzing next-generation DNA sequencing data. Genome Res 20:1297-1303. https://doi.org/ 10.1101/gr.107524.110.

64. Danecek P, Auton A, Abecasis G, Albers CA, Banks E, DePristo MA, Handsaker RE, Lunter G, Marth GT, Sherry ST, McVean G, Durbin R, 1000 Genomes Project Analysis Group. 2011. The variant call format and VCFtools. Bioinformatics 27:2156-2158. https://doi.org/10.1093/bioinformatics/btr330.

65. Cingolani P, Platts A, Wang LL, Coon M, Nguyen T, Wang L, Land SJ, Lu X, Ruden DM. 2012. A program for annotating and predicting the effects of single nucleotide polymorphisms, SnpEff: SNPs in the genome of Drosophila melanogaster strain w1118; iso-2; iso-3. Fly (Austin) 6:80-92. https://doi.org/10.4161/fly.19695. 